\title{
Efficient Support of IP Multicast in the Next Generation of GEO Satellites
}

\author{
Fethi Filali, Ghassane Aniba, and Walid Dabbous
}

\begin{abstract}
Satellites are expected to have an important role in providing the Internet protocol (IP) multicast service to complementing next-generation terrestrial networks.

In this paper, we focus on the deployment of IP multicast over the next generation of digital video broadcasting-based geosynchronous earth orbit satellites supporting multiple spot beams and on-board switching technologies. We propose a new encapsulation scheme optimized for IP multicast, which has two distinct modes enabling two alternative on-board switching approaches: the selfswitching and the label-switching. We also detail a set of mechanisms and protocols for ground stations, as well as for the on-board processor to allow an efficient multicast forwarding in this type of environment, while reducing the load of control and data messages in the satellite segment, and building efficient multicast delivery trees reaching only the spot beams containing at least one member of the corresponding multicast session.

To integrate satellite links in the terrestrial Internet, we present satellite multicast adaptation protocol (SMAP), a protocol which is implemented in satellite stations to process incoming protocol independent multicast-sparse mode (PIM-SM) messages sent by terrestrial nodes to the satellite system. SMAP helps to update the tables required for the mapping between IP packets and MPEG-2 data segments, their switching on board the satellite, and their filtering at the satellite receivers.
\end{abstract}

Index Terms-Digital video broadcasting via satellite (DVB-S), DVB-return channel via satellite (RCS), Internet protocol (IP), multicast geosynchronous earth orbit (GEO) satellites, multiple spot beams, on-board switching, protocol independent multicastsparse mode (PIM-SM) protocol.

\section{INTRODUCTION}

$\mathbf{S}$ ATELLITE communication technology has been developed for nearly 50 years. Over the past few years, the demand to use satellite devices to access the Internet is growing because satellite communication can deliver Internet services to consumers and institutions in remote areas of the world not covered by good terrestrial connectivity.

Geosynchronous earth orbit (GEO) transparent (bent-pipe) satellite systems have been an important element of telecommunications networks for many years serving, in particular, long

Manuscript received December 15, 2002; revised July 1, 2003 and September 20, 2003. The work of F. Filali and G. Aniba was performed while with the Institut National de Recherche en Informatique et en Automatique (INRIA).

F. Filali is with the Mobile Communications Department, Eurecom Institute, Sophia-Antipolis 06904, France (e-mail: filali@eurecom.fr).

G. Aniba is with the Institut National de la Recherche Scientifique, Energie, Matériaux et Télécommunications (INRS-EMT), Montreal, QC H5A 1K6, Canada (e-mail: ghassane@inrs-telecom.uquebec.ca).

W. Dabbous is with the Institut National de Recherche en Informatique et en Automatique (INRIA), Sophia-Antipolis FR-06902, France (e-mail: dabbous@sophia.inria.fr).

Digital Object Identifier 10.1109/JSAC.2003.819994 distance telephony and television broadcasting. The involvement of satellite in Internet protocol (IP) networks is a direct result of new trends in global telecommunications, where Internet traffic will hold a dominant share in the total network traffic. The large geographical coverage of the satellite footprint and its unique broadcasting capabilities, as well as its high-capacity channel combined with readily available Ka Band spectrum will retain satellite systems as an irreplaceable part of communications systems, despite the high cost and long development, and launching cycle of a satellite system.

To support high bandwidth applications, it is anticipated that the next-generation satellite communications systems will differ from the traditional systems by including intelligent functions in the on-board satellite, the use of ka-band and V-band, and the use of the spot beams technology. On-board processing (switching) will direct packets to each appropriate downlink spot beam. This will enable multiple uplink terminals (at different locations) to serve as feeds to the multicast receivers, thus providing (effectively) a space-bourne multicast overlay over the existing terrestrial Internet.

GEO satellite systems, in general, are well suited for multicast applications thanks to their broadcast capability, and especially, next-generation satellite systems, which lead to an efficient use of the downlink bandwidth given that multicast packets are expected to be forwarded by the satellite only to the spot beams that contain at least one member. However, there is no public and global framework for enabling IP multicast is this kind of system available in the open literature. Indeed, the system characteristics may handle different questions in the manner to use the IPs over satellite and in particular multicast protocols.

In this paper, we emphasize the support of IP multicast in the next generation of satellite systems that implement the digital video broadcasting (DVB) standard [6] to send data using MPEG-2 transport stream (TS) in the satellite segment. In this context, we study the issue of the encapsulation and the efficient segmentation of IP multicast packets into MPEG-2 TS data segments in order to allow the on-board satellite processor to switch received data segments to the appropriate spot beams based for example on a switching table containing the list of outgoing spot beams for each active session.

We also address the problem of adapting the independent multicast-sparse mode (PIM-SM) multicast routing protocol [16] used in the terrestrial network for the next generation of GEO satellite networks, which use the DVB via satellite (DVB-S) standard [6] in the downlink and the DVB-return channel via satellite (RCS) standard [5] in the uplink.

The remainder of this paper is organized as follows. We review, in Section II, the MPEG-2 standard and the main advances in IP data transmission over the DVB technology. Section III 
presents the architecture of the next generation of GEO satellite systems and its main components. In Section IV, we describe the multiprotocol encapsulation (MPE) scheme used in GEO transparent satellite systems and we outline its main limitations to not be used for the next generation of GEO satellite systems. We detail in Section V, our proposed IP-optimized encapsulation technique that could be used for two different on-board switching approaches, namely the label-switching and the self-switching, which are compared in Section VI. Section VII presents satellite multicast adaptation protocol (SMAP) a protocol which acts as an adaptation of the PIM-SM protocol for enabling the IP multicast delivery over the next generation of satellite systems using the DVB-RCS standard [5]. We conclude this paper in Section VIII.

\section{MPEG-2 AND IP OVER DVB}

The DVB system specified by the European Broadcast Union (EBU) is based on the cell-oriented packet transmission system defined by ISO/IEC 13818-1 MPEG-2 systems standard [11]. MPEG-2 systems standards provide the mean of multiplexing several types of multimedia information into one transport stream (TS) that can be transmitted over a variety of transmission media [17], [20].

Traditionally, an MPEG-2 TS contains packets of compressed video and audio data. The compression causes a variable data rate of each TV program because scenes with a lot of motion in the picture are encoded with a higher bit rate then scenes with less motion.

Within MPEG-2 TS, it is also possible to carry defined data containers in addition to the audio and video [20]. These data containers can be used to realize new data services or to carry IP datagrams.

Compressed data from a single source (i.e., audio, video, data, etc.) plus ancillary data needed for synchronization, identification, and characterization of the source information build up elementary streams (ESs). ESs are packetized into either constant-length or variable-length packets to form packetized elementary streams (PESs). Each PES packet consists of a header followed by stream data called the payload. PES packets from various elementary streams are combined to form a program.

Several programs combine to form the TS together with other descriptive data called program-specific information (PSI). PSI defines the program and its constituent parts.

As shown in Fig. 1, TS packets are 188 byte fixed-sized. Each TS consists of a TS header, followed optionally by ancillary data called the adaptation field, followed typically by some or all of the data from one PES packet. The TS header consists of the following fields.

- The header starts with the well-known synchronization byte, which has the bit pattern $0 \times 47(01000111)$.

- A set of 3 bits are used to indicate how the payload should be processed:

- the first flag indicates a transport error (transport error_indicator);

— the second flag indicates the start of a payload (payload_unit_start_indicator) (PUSI);

- the third flag indicates transport priority bit (transport_priority).

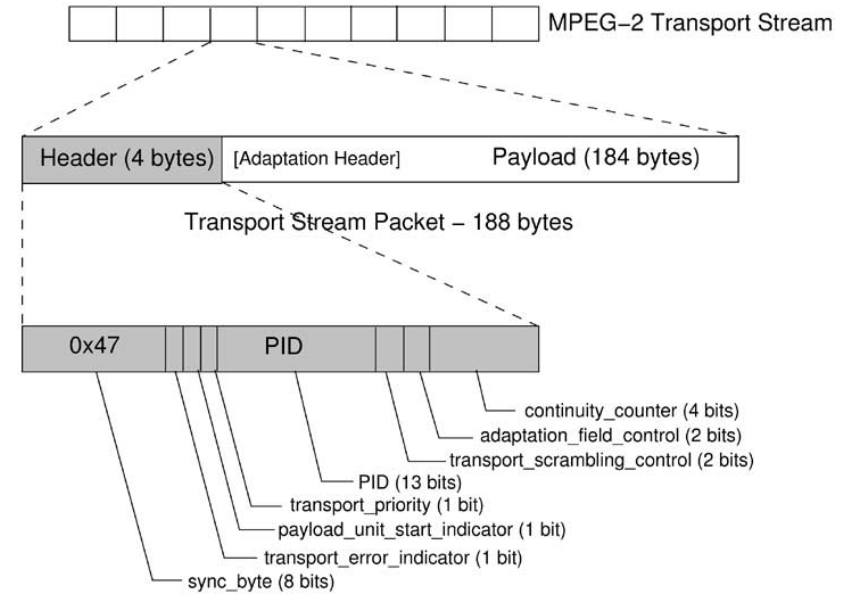

Fig. 1. Transport stream packet and header structure [2].

- The flags are followed by a 13-bit packet identifier (PID). This is used to uniquely identify the stream to which the packet belongs (e.g., PES packets corresponding to an ES generated by the multiplexer). The PID allows the receiver to differentiate the stream to which each received packet belongs. Some PID values are predefined and are used to indicate various streams of control information. A packet with an unknown PID, or one with a PID which is not required by the receiver, is silently discarded. The particular PID value of $0 \times 1 \mathrm{FFF}$ is reserved to indicate that the packet is a null packet (and is to be ignored by the receiver).

- The two scrambling control bits (transport_scrambling control) are used by conditional access procedures to encrypted the payload of some TS packets.

- Two adaptation field control bits (adaptation_field_ control) which may take four values:

1) 01-no adaptation field, payload only;

2) 10 - adaptation field only, no payload;

3) 11 - adaptation field followed by payload;

4) 00-RESERVED for future use.

- Finally, there is a half byte continuity counter (4 bits) (continuity_counter).

There are mainly five types of PSI streams [7], [10]: program association table (PAT), program map table (PMT), network information table (NIT), conditional access table (CAT), and digital storage medium command and control (DSM-CC). The decoder selects a desired program by extracting blocks, which have the required PID (the PIDs are described in the PAT and PMT blocks embedded in the transport stream). NIT specifies physical network parameters, while CAT carries information for scrambling. DSM-CC provides protocol and application program interface for user-to-network and user-to-user communications.

The DVB specification for data broadcasting [6] defines three different ways of inserting data into MPEG-2 transport stream.

1) Data packets can be encapsulated and carried inside the PES packets intended for video and audio streams. This method is referred as data streaming [6]. 
2) Data packets can be carried inside the section packets defined for system internal tables in DSM-CC. This method is called MPE [6].

3) An adaptation layer protocol can segment data packets directly into a sequence of cells. This method is called data piping [6].

All three methods involve a certain overhead, which stems from the header fields and the fact that IP datagrams usually do not come in multiples of 184 bytes. The total overhead for a transmission depends on the packet length distribution and the encapsulation method selected. The observed overhead for MPE is typically between $13 \%$ and $15 \%$ [2].

\section{NeXT-Generation SATEllite-TerRestrial Hybrid NETWORKS ARCHITECTURE}

\section{A. Architecture Description}

Prior to studying the IP multicast support standard over the next generation of GEO satellite networks, a general network architecture of this type of networks must be specified. A nextgeneration GEO satellite performs tasks like demodulation and decoding which allow signal recovery before retransmission. Since the signal is available at some points in baseband, other activities are also possible, such as routing, switching, etc. Most of such satellites in operation today or planned for deployment in the nearest future are characterized by the support of two interesting technologies.

- On-Board Switching (OBS): OBS provides a method of "network" switching on the satellite. It can be used to actively control signal routing. To cope with high network transfer rates, the switching of packets from an uplink to a different downlink spot beam needs to be done at a very high speed. There are two types of satellite switched network implementation 1) fully switched, where the satellite does all the processing and there is no or very little ground control and 2) intermediate frequency (IF) or radio frequency (RF) switching that involves electronically controlled RF/IF switches, which can be configured on a nearreal-time basis via ground control.

Note that our proposed schemes concerning the support of IP multicast for the next generation of GEO satellites do not depend on the technology used by the satellite to handle the switching.

- Spot Beams Technology: Instead of covering the whole footprint of a satellite by a global beam, the beam is divided into a number of spot beams. The benefits of spot beams are twofold: 1) the power requirements of user terminals are reduced, thereby permitting the use of smaller antennas in the ground segment and reducing cost and 2) the frequency between beams can be reused, thereby increasing the capacity of the space segment.

There are several ways of designing a return channel from the receivers to the satellite sender for multicast services, and many people believe that terrestrial return channels are the most costeffective and practical. Commonly proposed terrestrial return channels include public switched telephone network (PSTN), integrated services digital network (ISDN), and global system for mobile communication (GSM). However, there is a huge

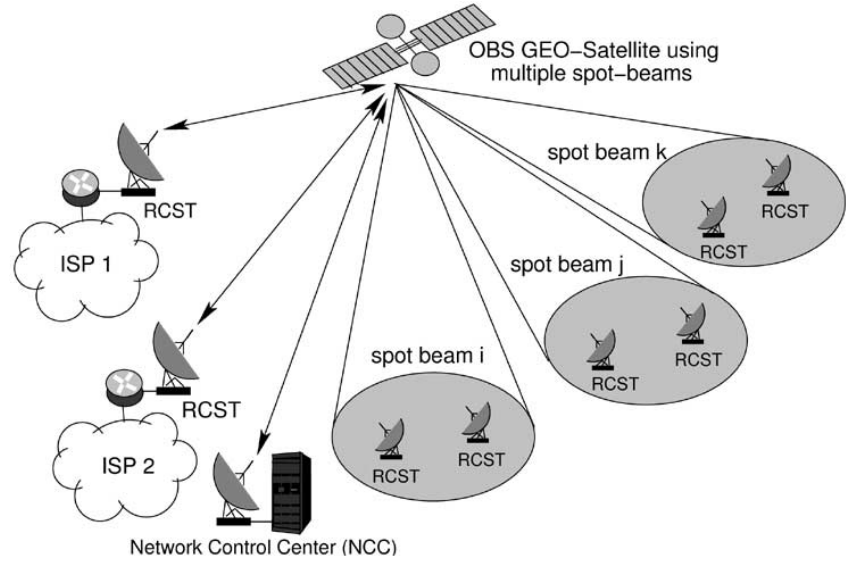

Fig. 2. Next-generation GEO satellite-terrestrial hybrid networks architecture characterized by the support of spot beams and on-board switching technologies.

worldwide interest in defining a return channel via satellite, and there are several reasons for this. First, the ordinary consumer does not want to be bothered by technical setups which require interconnections between the TV, PC, and telephone. A solution where all the technical equipment is concentrated within one box and without having to worry about blocked telephone lines etc., will certainly be appealing to many people. Another reason for choosing satellite services to provide interactivity is the increasing traffic on the terrestrial networks, which often results in blocking or reduced quality-of-service (QoS). Finally, there is an advantage, both for the users and the operators, that the forward and return channels are carried on the same medium. This enables better control of the QoS and the network management: terrestrial infrastructures are not always controlled by the same operator as for satellite, particularly when national borders are crossed.

Due to the recognized need for a specification in this area, the DVB technical module (DVB-TM) created an ad hoc group in early 1999 called DVB-RCS. As shown in Fig. 2, a nextgeneration GEO satellite-terrestrial network is expected to have two main entities.

- RCST $^{1}$ : Return channel via satellite terminal (RCST) is a feeder, with bidirectional link capability. In the remainder of this paper, we mean by RCST sender and RCST receiver the RCST which sends or receives the multicast packets depending on the writing context, respectively. An RCST may have both roles for different multicast sessions.

- Network control center (NCC): NCC is the core of the satellite network.

The NCC is in charge of the network control, which will include several RCSTs, but perhaps also several satellites, feeders, gateways, and even several networks. The RCST network to be managed is a multipoint-to-point structure, far more complex to administrate than the opposite, the point-to-multipoint structure. The NCC is, thus, in charge of the control of every RCST in the network, as well as the network as a whole. A terminal will $\log$ on after having received general information by

\footnotetext{
${ }^{1}$ In the DVB-S terminology, the satellite terminal is known as the satellite terminal (ST), a term which is used for transparent GEO satellites, and in the DVB-RCS [5] terminology is referred to as the RCST.
} 
listening to the forward link. The information given here is on the status of the network but, most importantly, the forward link provides the network clock reference (NCR). When the RCST has obtained synchronization with the NCR, it will use one of the designated slots (indicated in the forward channel) to issue a log-on request, in a slotted Aloha manner. If the terminal is successful with this request, the NCC will forward various tables containing general network and terminal-specific information. The specific information is about the necessary frequency, timing, and power-level corrections to be performed by the terminal before the transmission starts. These tables will also indicate the resources allocated for the terminal, and it is possible to ask for different services or increased capacity during transmission. The NCC has the possibility, with certain intervals, to correct the transmission parameters of the RCST and, if something goes wrong during transmission, the NCC shall also have the possibility of forcing log-off from the RCST. The continuous signaling from the NCC is provided according to MPEG-2 system information (SI) [17].

Using the common signaling channel (CSC) message which is defined in the DVB-RCS standard, the RCSTs acquire all information needed for the data transmission on the satellite segment. This information includes the PID used for sending data, as well as the PID reserved for control messages (CTRL_MNGM_PID) between the RCSTs and the NCC.

\section{B. Network Protocol Stack}

In Fig. 3, we show an example of the protocol stack on the RCST and the satellite in the case of Ethernet terrestrial connection. We assume that the system integrates two existing satellite transmission standards: DVB-RCS [5] and DVB-S [6]. The later standard is used at the satellite uplink, whereas the former is used at the satellite downlink. Note also that both of them are also used in GEO transparent satellite systems without any regeneration on board. The system combines these two standards into a single regenerative multispot satellite system allowing for full cross-connectivity between the different uplink and downlink beams. We also assume the use of MPEG-2 standard on top of DVB.

As shown in Fig. 3, the uplink is assumed to be compliant with the DVB-RCS standard. This fact will allow users to use standard RCST stations, which will are expected to be widespread and relatively inexpensive in the future thanks to the standardization effort of terminal manufacturers and broadcast satellite operators. The downlink will be fully compliant with the DVB-S standard, including all the possible convolutional rates. This will allow users to take advantage of the economies of scale and the performance of standard commercial DVB-S receivers, which are widespread across Europe today. A key feature of the system will be the capacity to route data on any of the uplink coverage footprints on to any combination of downlink coverage footprints; the system will implement full cross-connectivity between uplink and downlink footprints. In order to accomplish all this, contributions from all DVB-RCS compliant uplink users must be demultiplexed, demodulated, and decoded and then switched and remultiplexed into the DVB-S compliant downlink data streams as required by users. On-board switching and multiplexing will take place in accordance with a dynamic

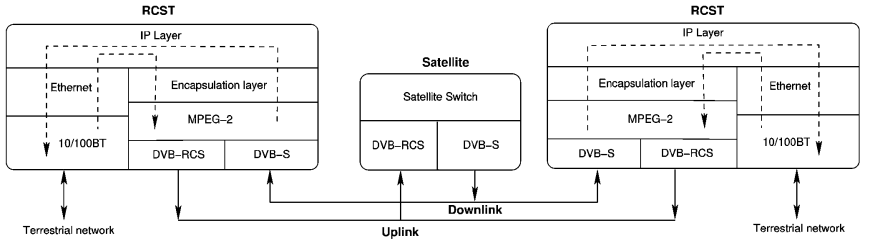

Fig. 3. Protocol stack.

multiplexer table. Each downlink has associated with it a multiplexing table. It will be possible to reconfigure this table very quickly through a signaling channel allowing very fast circuit switching at packet level onboard.

As we can see from Fig. 3, the encapsulation layer takes place between the MPEG layer and the IP layer. Its role at an RCST sender is to add a specific header to the IP packet received from the upper IP layer, and send the new packet to the lower layer (MPEG-2 layer). At an RCST receiver, this layer reassemble the received payloads from the MPEG-2 layer into one encapsulation layer packet and removes the header added by the RCST sender from and sends the resulting IP packet to the IP layer.

The satellite protocol stack consists only in two layers: the link layer which is responsible for the processing of incoming and outgoing data segments and the DVB layer (DVB-S and DVB-RCS), which represents the physical layer. The on-board processor (OBP) switches the received data segments to the destination ports (spot beams).

\section{MPE ENCAPSULATION SCHEME}

As we have outlined in Section II, the MPE scheme is one of the three methods which are described by the DVB specification for data broadcasting [6] to carry voice, audio, and data in MPEG-2 transport segments. The data containers (called datagram sections) of the MPE method are expected to be optimized for carrying IP datagrams.

In this section, we first describe the MPE encapsulation scheme, and then we outline its main limitations to be applied in the next generation of GEO satellite systems.

\section{A. Scheme Description}

The MPE specification of DVB uses private sections for the transport of IP datagrams and uses an encapsulation, which is closely tailored after the IEEE local area network/metropolitan area network (LAN/MAN) standards. Data packets are encapsulated in datagram_sections, which are compliant with the DSM-CC section for private data [6]. This encapsulation makes use of a medium access control (MAC) level device address. The address format conforms to the ISO/IEEE standards for LAN/MAN.

We illustrate, in Fig. 4, how IP datagrams are encapsulated within DVB-MPE datagram sections and are then segmented into MPEG transport packets. IP datagrams must be fragmented at the IP layer such that they do not exceed the specified maximum transfer unit (MTU) (which is equal to 1500 bytes for Ethernet) for the payload portion of the DVB-MPE datagram section.

Devices delivering IP datagrams within DVB MPE datagram sections must segment datagrams such that they do not exceed a 


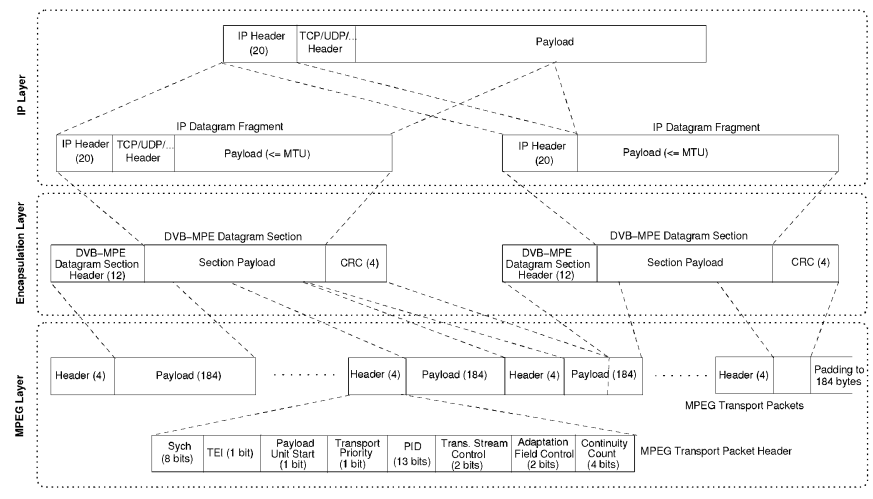

Fig. 4. Carrying IP packets into MPEG segments using the MPE encapsulation approach.

specific size called, the MTU of the network. IP datagrams can be as large 65536 bytes $^{2}$ in length. Therefore, the source device must use IP layer fragmentation to breakup the large IP datagrams no larger than the MTU before passing them to the data link layer, where they will be encapsulated within DVB-MPE datagram sections. Likewise, when the DVB-MPE datagram sections are received by the decoder, the IP fragments will be passed to the IP layer software for reassembly into a complete IP datagram.

\section{B. Reasons to not use MPE}

We identify three reasons the MPE scheme as presented above cannot fulfill the requirement of these systems for three major reasons. First, the MPE scheme is not optimized for transporting IP packets given that not all the header fields added to each IP packet are required to deliver IP packets to the destinations. Second, when using the MPE scheme, the on-board satellite processor will not be able to determine the session to which the received MPEG data segment belongs and then it is not able to forward it to the correct list of outgoing ports (spot beams). Third, in the context of multicast delivery, the filtering in the MPE layer is based only on the MAC destination address, which is a standard mapping of the IP destination address [4]. Or, a multicast session is identified by the pair (source address, group address), for example when using the shortest-path tree (SPT) mode of PIM-SM [16] or for the single-source multicast (SSM) service model [15]. It is, therefore, impossible to handle the filtering at the lower layer (link layer), which makes the upper layer (IP layer) more complex and forces it to filter more packets.

Note that the IETF proposed "IP over DVB" working group, ${ }^{3}$ is designing a new encapsulation scheme for transmitting IP packets over DVB medium [3], [13] however, our work focuses particularly on the IP multicast support over the new generation of GEO satellite systems.

To allow the RCSTs to filter incoming packets and to help the on-board satellite to switch incoming MPEG-2 data segments to the appropriate spots, we propose a new encapsulation layer called IP-optimized encapsulation scheme, which will replace the MPE layer used in the MPE method.

\footnotetext{
${ }^{2}$ Decoders may limit the maximum IP datagram size due to internal buffering constraints. Note also that in the maximum payload for DSM-CC is 4086 bytes.

${ }^{3} \mathrm{http}: / / w w w . e r g . a b d n . a c . u k / u s e r s / g o r r y / i p-d v b /$
}

\section{IP-OPTIMIZED ENCAPSULATION SCHEME}

The next generation of GEO satellites are expected to use an OBP with multiple spot beams. We study the IP packet delivery in this type of satellites and especially for multicast communication. As we have outlined earlier, the satellite receives MPEG data segments that should be processed on board and switched to the correct outgoing ports. Therefore, each data segment should contain an information that helps the OBP to handle the switching. The MPE encapsulation scheme detailed in Section IV does not provide this kind of information and so it is not adequate for the new generation of GEO satellite systems.

Our goal is to design a new encapsulation scheme (instead of using the MPE method) between the IP level, and the MPEG2 level that allows the switching in the on-board satellite processor, as well as the efficient filtering of multicast packets at the RCST receivers. The design of this scheme should take into account the required sharing tasks between the satellite and the ground stations to ensure the IP multicast delivery over the next generation of satellite-terrestrial networks. The IP-optimized encapsulation scheme has two distinct modes that allow to use two different switching approaches.

- The label-switching approach, is the fact of switching the MPEG data segments on the satellite based on an on-board switching table.

- The self-switching approach, is the fact of switching the MPEG data segments on board the satellite based on a label added by the RCST sender to the header of each data segment.

Each approach has to provide three main functionalities.

- Mapping: This functionality deals with the mapping of each IP packet at the RCST sender to a set of MPEG data segments and the information to add to the IP packet header in order to allow the switching on board the satellite. This function specifies also how the data segments should be reassembled at the RCST receiver.

- Switching: This is a functionality of the on-board satellite processor which describes the procedure used to switch the incoming MPEG data segments to the appropriate outgoing ports (spot beams). The switching function should not depend on the technology used by the satellite to handle the switching: fully switched or IF/RF switching.

- Filtering: This functionality deals with the filtering procedure of the incoming MPEG segments at the RCST receiver. The lower layer (link layer) should forward to the upper layer (network layer) only the segments that belong to an IP packet which should be received by the terminal.

The mapping procedure should provide the required information to the switching and filtering functions. We detail in the following section the three functionalities for each switching approach (label-switching or self-switching).

\section{A. Label-Switching Approach}

The label-switching consists on the use of a switching table maintained by the on-board satellite to forward the incoming data segments to the correct list of spot beams. This type of 


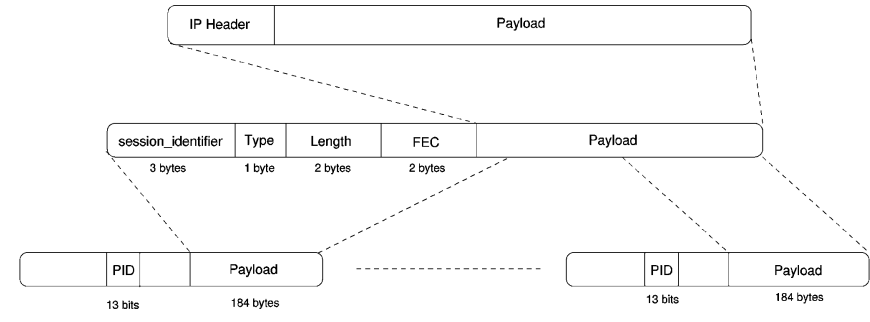

Fig. 5. IP-optimized encapsulation scheme mode for the label-switching and on-board switching approach.

routing corresponds to the method used in the classical IP terrestrial networks. Indeed, routers use routing tables to switch the received IP packets to the correct outgoing interface(s). For these reasons, we call this approach the "label-switching" approach.

1) Mapping: The switching on board the satellite based on the label-switching approach requires that the identifier of the packet should be added by the RCST sender on each data segment. This could be possible through the direct mapping between IP addresses and PID identifiers in the MPEG-2 TS data segment header [18], which allows the use of the existing DVB cards without any modification or improvement. However, the PID is coded in 13 bits allowing only $2^{13}$ simultaneous connections to be mapped, which is not enough comparing with the available IP address growing. Moreover, different IP addresses will be mapped to the same PID, which does not provide an efficient switching in the on-board satellite processor and makes impossible the efficient filtering at the MPEG layer of the RCST destination. On the other hand, current RCSTs can only filter, with a hardware implementation, at most 32 simultaneous PIDs. This problem may be avoided using a software implementation of the data segments filtering module.

To overcome these problems, we propose a new encapsulation scheme that can be used instead of the digital storage media command and control (DSM-CC) [19] scheme used in MPE. We suggest an encapsulation method dedicated for GEO satellites with OBP and multispots satellite. As shown in Fig. 5, a new header is added to each IP packet. This header consists on four fields:

- session_identifier: the identifier of a multicast session;

- type: is the type of the upper protocol;

- length: contains on the length of the IP packet;

- FEC: included in order to guarantee the good transmission of the various fields of the new header.

The new packet, is segmented to several payload data which is equal to 184 bytes. A standard MPEG header of 4 bytes is added at the MPEG layer to each data payload to compose a MPEG-2 TS. The data segments will be then sent successively to the satellite interface.

Note that the session identifier field corresponds to the first 3 bytes of the payload of the first MPEG-2 TS and that this data segment can be identified at the satellite based on the PUSI flag of the MPEG-2 header. In addition, given that the session_identifier field is coded in 3 bytes, this encapsulation allows the mapping of $2^{24}$ IP addresses simultaneously.
TABLE I

MAPPING TABLE ON THE RCST SENDER FOR THE LABEL-SWITCHING APPROACH

\begin{tabular}{c|c}
\hline (@source, @Destination) & session_identifier \\
\hline \hline$@ s_{1}, @ d_{1}$ & ox000001 \\
\hline$@ s_{1}, @ d_{2}$ & ox000002 \\
\hline$@ s_{3}, @ d_{1}$ & ox000003 \\
\hline$\ldots$ & $\ldots$ \\
\hline$@ s_{3}, @ d_{2}$ & oxFFFFFE \\
\hline
\end{tabular}

TABLE II

PERMANENT Switching TABLE ON THE SATELlite

\begin{tabular}{c|c}
\hline session_identifier & Spots \\
\hline \hline ox000001 & 1,2 \\
\hline ox0000E2 & $4,5,9$ \\
\hline.. & $\ldots$ \\
\hline ox0FE002 & $11,14,18,20$ \\
\hline
\end{tabular}

TABLE III

TEMPorary Switching TABLE ON THE SATELLITE

\begin{tabular}{c|c|c}
\hline Input Spot & PID & Output Spots \\
\hline \hline 1 & pid 2 & 1,2 \\
\hline 2 & pid 7 & $4,5,9$ \\
\hline$\ldots$ & $\ldots$ & $\ldots$ \\
\hline 32 & pid 20 & $11,14,18,20$ \\
\hline
\end{tabular}

Each RCST sender maintains a mapping table ${ }^{4}$ that gives the mapping between the IP packet identification and the session_identifier field. For example, Table I shows the mapping between IP multicast packets and the session_identifier. In this case, the identifier of the IP multicast packet is the pair (@source, @Destination).

When receiving an IP packet from the upper layer to send through the satellite interface, the encapsulation layer of the RCST sender determines the corresponding value of the session_identifier from the mapping table to be included in the header.

2) Switching: When receiving a data segment from an RCST sender, the satellite should determine the list of spot beams to which it has to forward the received segment.

In order to handle the switching on board the satellite, it is necessary that the OBP keeps and updates two main switching tables. The first one is the permanent switching table shown in Table II, which provides for each session_identifier the list of destination spots. This table is updated by the NCC which periodically sends a message to the satellite that specifies for each active session (a specific session_identifier) the list of destination spots. The same message is also broadcasted to all the RCSTs in order to update their own tables. The proposed protocol to handle these messages will be described in Section VII.

The second table (temporary switching table), shown in Table III, gives for each input port the PID and the list of destination spot beams of the current received data segment. This table is updated by the satellite when it receives the first MPEG-2 data

\footnotetext{
${ }^{4}$ We describe later how this table is updated and how all mapping tables are synchronized between the RCSTs.
} 


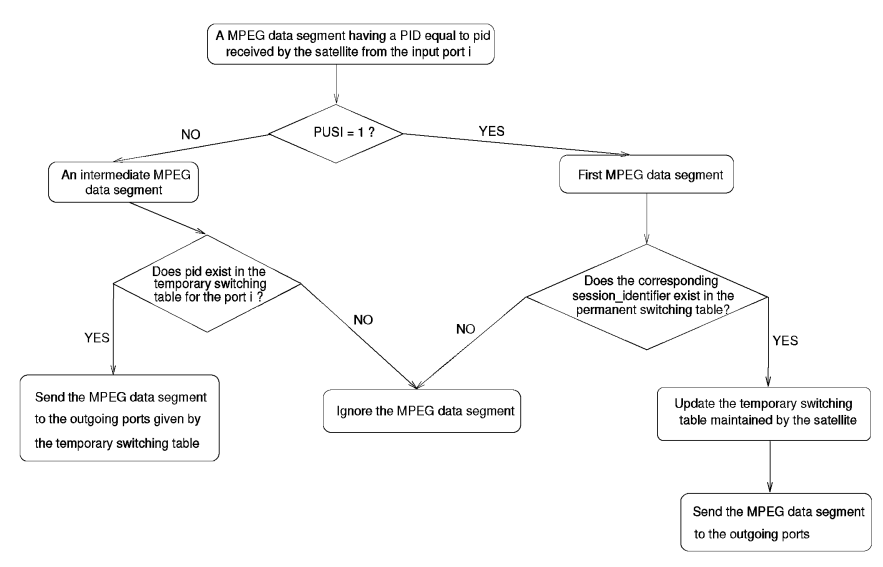

Fig. 6. Algorithm of processing incoming MPEG-2 data segments and updating the temporary switching table.

TABLE IV

SUBSCRIPTION TABLE ON THE RCST RECEIVER

\begin{tabular}{c|c|c}
\hline session_identifier & (@source,@IP Destination) & State \\
\hline \hline ox000002 & $@ s_{1}, @ d_{1}$ & Standby \\
\hline oxFFFFFE & $@ s_{1}, @ d_{2}$ & Available \\
\hline ox000001 & $@ s_{2}, @ d_{1}$ & Active \\
\hline$\ldots$ & $\ldots$ & $\ldots$ \\
\hline ox000004 & $@ s_{3}, @ d_{2}$ & Online \\
\hline
\end{tabular}

segment $^{5}$ that belongs to a new IP packet from an input port. From this first data segment, it determines from the payload (the first 3 bytes) the session_identifier value of the packet and the PID and copies the corresponding list of destination spots from the second column of the permanent switching table (Table II) to the third column of the temporary switching table (Table III). The flowchart of the algorithm used to update this table is shown in Fig. 6.

It can be noted that the first table is quite permanent, while the second one may vary on each reception of a data segment that belongs to a new IP packet (e.g., has a different PID or a PUSI flag bit set to 1).

3) Filtering: Each RCST receiver maintains a table at the encapsulation layer that we call, the subscription table as shown in Table IV. This table consists in three columns. For each session_identifier value, given in the first column, it indicates the IP identifier [for example, (@IP source, @IP destination) pair for IP multicast connections] of the corresponding IP packet in the second column. As we have outlined earlier, this mapping is done by the NCC for all active sessions and it is periodically broadcasted to all the RCSTs. The third column of the subscription table specifies the session state, which is very useful to reduce the number of data segments sent from the MPEG layer to the encapsulation layer at the RCST receivers and so the number of IP packets sent to the network layer. As we will explain later, this column is set by the RCST receiver modifies the state of the multicast sessions according to the PIM-SM messages received from the downstream members and the DVB messages broadcasted by the NCC.

${ }^{5}$ Recall that based on the PUSI flag in the MPEG2-TS, the satellite can detect the first data segment of an IP packet.
TABLE $\mathrm{V}$

Filtering TABLE of THE PIDS MAINTAINED AT THE LINK LAYER (MPEG-2 LEVEL) OF THE RCST

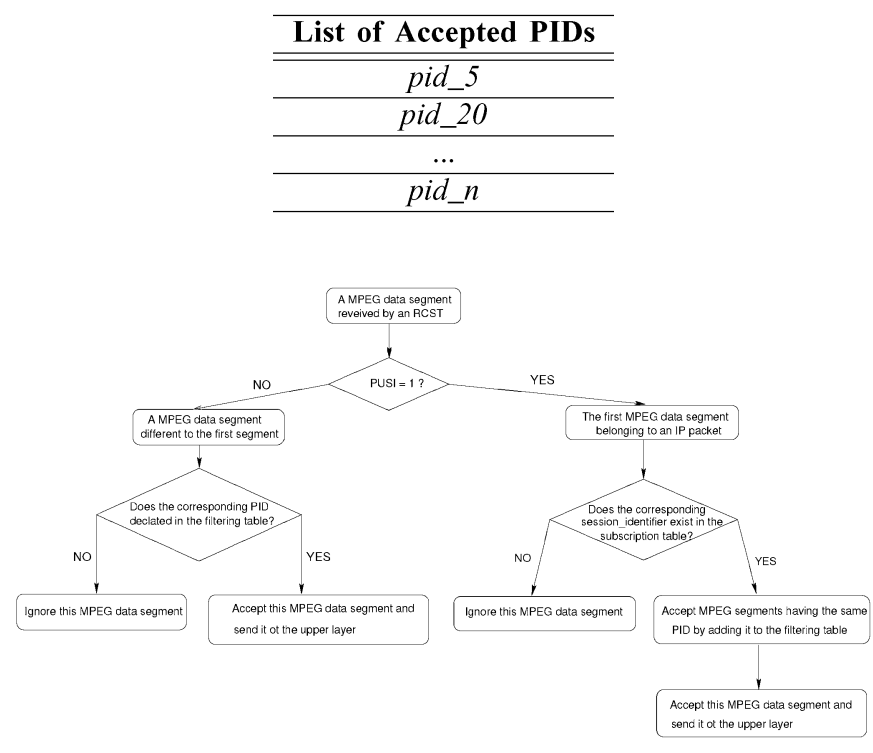

Fig. 7. Filtering algorithm at the MPEG layer of the RCST receivers.

The field "state" could have the following values.

- Standby: informs that this session is accessible (i.e., there is at least one source sending to the corresponding multicast group) but there is no subscriber for this session downstream to all RCSTs. This state is the initial one of each new entry created by the RCST when receiving an updated information from the NCC.

- Available: informs that this session is active but all the members belong to spot-beams other than that to which the RCST belongs.

- Active: means that this session is accessible via the satellite network in the spot beam of the RCST. In other words, this RCST is receiving data from this session but there is no member downstream to this RCST.

- Online: informs that this session is requested and the received data segments should be forwarded to upper layers in order to be reassembled and sent to the terrestrial interfaces, where there are downstream members.

To allow the filtering of incoming data segments at the MPEG layer, each RCST maintains at the MPEG level a filtering table containing the list of current accepted PIDs (e.g., those having the state set to "online") as shown in Table V.

A simple protocol between the network, the encapsulation, and the link (MPEG) layer could be designed to update the third column of the subscription table and the filtering table. The detail of this protocol is out of the scope of the research work described in this paper.

The flowchart of the filtering algorithm used to filter the incoming data segments at the MPEG layer and to update the list of accepted PIDs is given in Fig. 7. As we can see, a data segment will be sent to the upper layer only if it concerns a session which belongs to the set of active sessions with a state "online" in the subscription table of Table IV. In this case, the PID will be added to the filtering table if it has not already been added. 


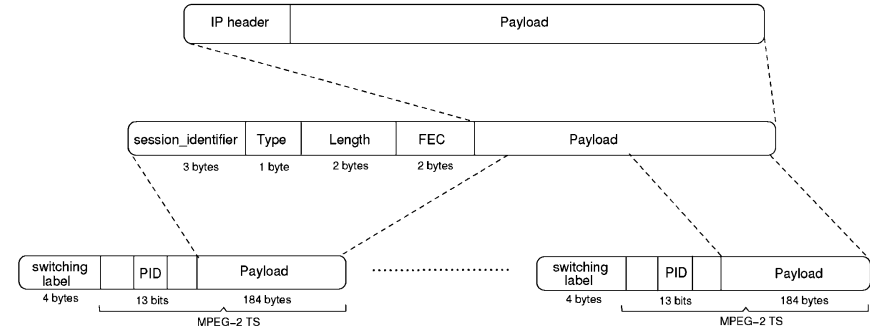

Fig. 8. The IP-optimized encapsulation scheme mode for the self-switching approach.

TABLE VI

The MAPPING TABLE ON THE RCST SENDER FOR THE SELF-SWITCHING APPROACH

\begin{tabular}{c|c|c}
\hline (@source, @Destination) & session_identifier & switching_label \\
\hline \hline$@ s_{1}, @ d_{1}$ & ox00001E & ox00403F10 \\
\hline$@ s_{2}, @ d_{1}$ & ox1F00A2 & ox00400410 \\
\hline$@ s_{1}, @ d_{3}$ & ox00B0C3 & ox90403180 \\
\hline$\ldots$ & $\ldots$ & $\ldots$ \\
\hline$@ s_{n}, @ d_{m}$ & oxA0F270 & ox00010001 \\
\hline
\end{tabular}

\section{B. Self-Switching Approach}

Recall that the self-switching approach consists in adding specific information to each MPEG-2 data segment in order to allow the on-board satellite processor to switch it to the correct list of the outgoing destination ports without using an on-board switching table as the label-switching approach does.

1) Mapping: The mapping for the self-switching is the same as that of the label-switching, except that we add a new field called switching_label to each MPEG data segment in order to encode the list of destination ports of the corresponding session. The size of this field depends on the number of spot beams in the satellite system. For example, with a field of 4 bytes, it is possible to support the self-switching approach for 32 spot beams. Each bit in the "switching_label" field concerns a single outgoing port on satellite linked to a specific spot beam. This field will be removed by the satellite before forwarding it to the outgoing ports in order to allow the use of current MPEG-2 drivers and to reduce the overhead in the satellite downlink.

As shown in Fig. 8, we still using the field session_identifier that we have proposed for the label-switching approach detailed in the previous section.

Other fields have the same role as when using the labelswitching approach. As it is shown in Table VI, the mapping table on the RCST sender, will be extended by adding the switching_label field.

Before sending each data segment, the RCST sender should add the corresponding switching_label and other fields. As we will explain later, the value of the switching label is determined from the information multicasted by the NCC, which gives the list of destination spots of each active session identified by its session_identifier as for the case of the label-switching approach.

2) Switching: The switching on the on-board satellite processor is based on the switching_label field included in each data segment. Therefore, there is no need to keep a switching table as for the case of the label-switching approach.

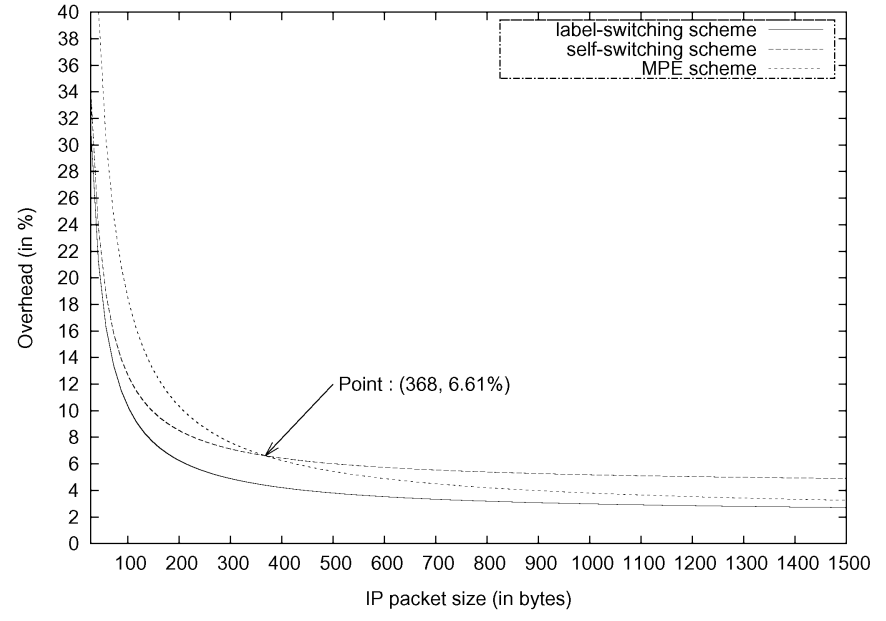

Fig. 9. Overhead comparison of self-switching, label-switching, and MPE approaches.

For each incoming data segment, the satellite has to read bit-per-bit the switching label inserted by the RCST sender in the head of each MPEG-2 header. When the bit number $i$ is set to 1 , it means that the satellite should forward the data segment to the port number $i$.

3) Filtering: The filtering procedure at the MPEG layer of the RCST Receiver is exactly the same as the case of the labelswitching approach given that the formats of the packet that arrives to the RCST receiver are similar for both approaches (see Section V-A3). Indeed, as we have pointed out earlier, the satellite removes the switching_label field before forwarding each data segment to the corresponding outgoing ports.

\section{ON-BOARD SWITCHING APPROACHES COMPARISON}

\section{A. Overhead}

Let $S$ be the size of the IP packet. We define the overhead of each approach as the amount of header added both by the encapsulation scheme and the MPEG layer over the size of the IP packet. Hence, the overhead of the three approaches (labelswitching, self-switching, and MPE) is computed as follows.

- The Label-Switching Approach: There are 8 bytes added by the encapsulation layer to the IP packet and 4 bytes added by the MPEG layer for each MPEG-2 payload (184 bytes). The overhead $f(S)$ of the label-switching approach is, therefore, equal to $(((S+8 / 184) * 4+8) / S)$.

- The Self-Switching Approach: There are 8 bytes added by the encapsulation layer to the IP packet, 4 bytes added by the MPEG layer for each MPEG-2 payload (184 bytes), and 4 bytes of the switching_label field to each MPEG-2 data segment. The overhead $g(S)$ of the self-switching approach is, therefore, equal to $(((S+8 / 184) * 8+8) / S)$.

- The MPE Approach: There are 16 bytes added by the encapsulation layer to the IP packet, and 4 bytes added by the MPEG layer for each MPEG-2 payload (184 bytes). The overhead $h(S)$ of the MPE approach is, therefore, equal to $(((S+16 / 184) * 4+16) / S)$.

We show in Fig. 9, the variation of the overhead of the labelswitching, the self-switching, and the MPE approaches in func- 
tion of the IP packet size. ${ }^{6}$ As we can see, the overhead of MPE and self-switching approaches decreases with the IP packet size, while remaining less than that added by the label-switching scheme which has an overhead less than $4 \%$ when the IP packet size is more than 400 bytes. Furthermore, the MPE approach adds more overhead than the self-switching approach when the IP packet size is less than 368 bytes. For an IP packet size equal to 368 bytes, the overhead is equal to $6.61 \%$ for both approaches. Note that even that the MPE provides better (less) overhead than the self-switching scheme when that IP packet size is more than 368 bytes, it could not be used to enable the on-board switching.

\section{B. Complexity}

Contrary to the label-switching approach, where the satellite needs to maintain and update an on-board switching table, for the self-switching approach the design of the GEO satellite is less complex and it is much more easier to process incoming MPEG-2 data segments given that it is based on the switching label field already included in the data segment. Furthermore, the switching to the outgoing spot beams could be implemented in a remote programmable switch for the self-switching approach. However, the RCSTs are more complex for the selfswitching approach because they need to compute the value of the switching_label field for each IP packet to be sent to the satellite interface into MPEG data segments.

According to Section VI-A, the overhead $g(S)$ of the self-switching scheme could be expressed as $g(S)=2 * f(S)+(8 / S)$, where $g(S)$ is the overhead of the label-switching scheme. When the packet size increases the value of $g(S)$ is approximatively equal to $2 * f(S)$. However, in the satellite downlink, both approaches consume the same resources (have the same overhead) given that the switching-label field is removed from the MPEG-2 data segment before being forwarded to the satellite receivers when using the self-switching approach.

We show in Table VII, the complexity of the label-switching and the self-switching approaches. From this comparative table, we can conclude that from the economic point of view, we can say that the label-switching approach is bandwidth conservative in the satellite uplink than the self-switching approach and from the technical point of view, the self-switching approach is simpler than the label-switching approach.

\section{SMAP PROTOCOL}

In this section, we describe SMAP that enhances the forwarding of the multicast packets on the satellite. It is a convergence signaling protocol between the multicast routing protocol used in the terrestrial network [PIM-SM, distance vector multicast routing protocol (DVMRP), etc.] and the DVB signaling messages used in the satellite segment. The main task of SMAP is to update the mapping, switching, and filtering tables used to enable the on-board switching in the GEO satellite.

In this section, we first describe a new DVB descriptor which will be used by the NCC to announce the multicast active ses-

\footnotetext{
${ }^{6}$ The minimum IP packet size is equal to 28 bytes ( 20 bytes of the IP header and 8 bytes of the link layer control (LLC) header).
}

TABLE VII

LABEL-Switching Versus SElF-Switching APPROACH

\begin{tabular}{c|c|c}
\hline & label-switching & self-switching \\
\hline \hline Complexity of the RCST & medium & high \\
\hline Complexity of the Satellite & high & little low \\
\hline Expected Terminal Cost & medium & little high \\
\hline Feasibility & yes & yes \\
\hline Compatibility with MPE DVB cards & no & no \\
\hline
\end{tabular}

TABLE VIII

SESSION DESCRIPTOR FORMAT

\begin{tabular}{l|r}
\hline Syntax & Number of bits \\
\hline \hline Session_Descriptor() \{ & \\
\hline descriptor_tag & 8 \\
\hline descriptor_length & 8 \\
\hline session_identifier & 24 \\
\hline switching_label & 32 \\
\hline address_type & 1 \\
\hline source_address & $32 / 128$ \\
\hline group_address & $32 / 128$ \\
\hline ? & \\
\hline
\end{tabular}

sions to the RCSTs. Then, we discuss how the RCST senders announce new sessions to the NCC and how the RCST members join and leave the multicast sessions. Finally, we detail the processing of PIM-SM messages received by the RCSTs in order to optimize the multicast delivery in the satellite segment.

\section{A. Multicast Session Descriptor}

Our approaches described earlier, namely the label-switching and the self-switching use the information broadcasted from the NCC to maintain and update the subscription table on the RCST receiver (see Table IV) and the mapping table on the RCST sender (see Tables I and VI). In the DVB standard systems, the NCC periodically sends signaling tables that manage some functionalities such as the PIDs allocation, the frequency usage, etc. Each information is described in a specific descriptor. In order to provide an efficient support of multicast applications in the new generation of satellite systems, we propose to add a new descriptor to periodically announce the multicast session and to send the information required by the different components of the system (the OBP, the NCC, and the RCSTs).

We call our new descriptor session_descriptor which is shown in Table VIII.

As we can see, there are seven fields, where every one can be used either by the satellite, or by the RCSTs, or by both of them. These fields are the following.

- session_identifier: As we have explained above, it is an identifier which identifies the multicast session of the descriptor.

- switching_label: is used by the satellite in case of the labelswitching approach in order to build the switching table of Table II and it is used by the RCST senders in the case of the self-switching approach in order to insert this label in the switching_label field at the head of each MPEG data segment.

- address_type: indicates which IP version is used; it is equal to 0 when using IPv4, and to 1 for IPv6. 
TABLE IX

NEW TYPES OF IE

\begin{tabular}{c|c|c}
\hline Id. & IE type & Usage \\
\hline \hline 0x0E & New Session & RCST Sender announces a new session \\
\hline 0x0F & Join / Leave Session & RCST Receiver joins/leaves sessions \\
\hline
\end{tabular}

- source_address and group_address: are the multicast group IP address and the multicast sender unicast address, respectively; they may be encoded in 4 or 16 bytes depending on the IP version given by the address_type field.

The session_descriptor is included in each Bouquet allocation table (BAT) [5]-[7] according to the content of the data unit labeling method (DULM) messages.

The BAT is an optional table within the DVB standard. We choose this table to enable the use of our approach in the satellite systems because it is the only table in the standard which informs about the general state of the satellite network.

The NCC keeps all information about the active multicast sessions and periodically informs the RCSTs about the active sessions using the session_descriptor. When there is a change in the state of a given session, ${ }^{7}$ it immediately broadcasts the corresponding session_descriptor (see Table VIII), within the BAT [5], [7], with a PID equal to $0 \times 0011$ according to the DVB standard [7], [9].

When receiving the BAT table from the NCC, an RCST receiver checks, in addition to the standard fields defined in the DVB-RCS standard [5], the source_address, group_address, and switching_label fields, and it updates its subscription table (Table IV).

\section{B. Using DULM Messages}

To handle signaling messages between the NCC and the RCSTs, we use two new information elements (IEs) types [5], which are defined in Table IX. The first one (id $=0 \times 0 \mathrm{E}$ ) will be used by the RCSTs senders, and the second one (id $=0 \times 0 \mathrm{~F}$ ) by the RCSTs receivers. These messages are sent using a PID equal to CTRL_MNGM_PID, which is allowed to be used by every RCST in the connection phase.

Following the standard specifications of DULM messages [5], we propose a specific format for both messages.

In Fig. 10, we show the format of the DULM new session message. We added three main new fields to those already defined in the DVB-RCS [5] standard:

- field @ Type defines the type of the IP addresses, equal to 0 for IPv4 and 1 for IPv6;

- field Private Data is a reserved filed for specific utilization for each operator;

- fields source_address_n and group_address_n defines the addresses that determine the new flow of session $n$.

Thanks to this message, every RCST sender informs the NCC about the new active sessions having the source behind him. Periodically, the RCST sender transmits a DULM new session message as shown in Fig. 10 toward the NCC in order to inform it about the current multicast active sessions. When receiving a DULM new session message, the NCC updates its multicast

\footnotetext{
${ }^{7}$ For example, a change on the switching_label value.
}

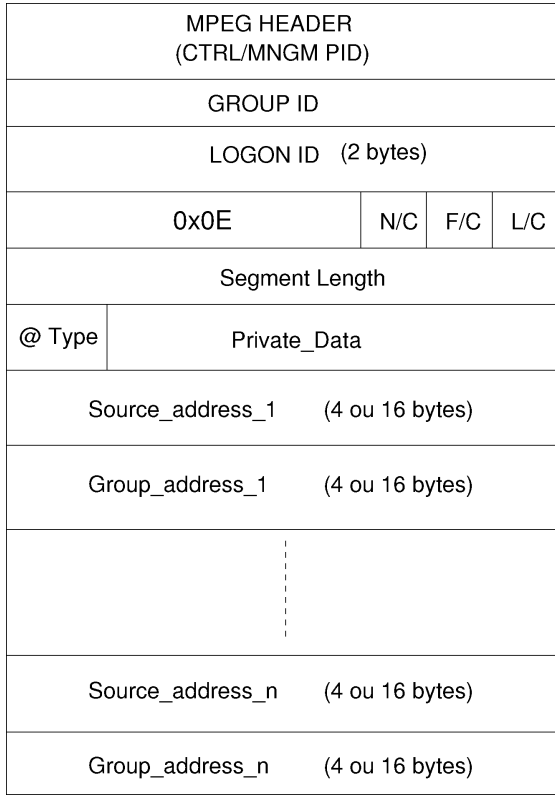

Fig. 10. Format of the DULM new session message.

\begin{tabular}{|c|c|c|c|c|}
\hline \multicolumn{5}{|c|}{$\begin{array}{l}\text { MPEG HEADER } \\
\text { (CTRL/MNGM PID) }\end{array}$} \\
\hline \multicolumn{5}{|c|}{ GROUP ID } \\
\hline \multicolumn{5}{|c|}{$\begin{array}{l}\text { LOGON ID } \\
\text { (2 bytes) }\end{array}$} \\
\hline & $0 \times 0 F$ & $\mathrm{~N} / \mathrm{C}$ & $\mathrm{F} / \mathrm{C}$ & L/C \\
\hline \multicolumn{5}{|c|}{ Segment Length } \\
\hline$J / L$ & \multicolumn{4}{|c|}{ Private_Data } \\
\hline \multicolumn{5}{|c|}{ session_identifier_1 } \\
\hline \multicolumn{5}{|c|}{ session_identifier_2 } \\
\hline \multicolumn{5}{|c|}{ session_identifier_j } \\
\hline \multicolumn{5}{|c|}{ session_identifier_n } \\
\hline
\end{tabular}

Fig. 11. Format of the DULM join/leave session message.

sessions table (MST), which describes the set of all multicast active sessions sending to the satellite network. In addition, the NCC assigns a new identifier session_identifier and adds the session_descriptor (see Table VIII) to the the BAT to be broadcasted to all the RCSTs.

In Fig. 11, we show the format of the DULM join/leave session message. There are three new fields added to those already defined in the DVB-RCS [5] standard:

- the bit $J / L$ (join/leave) specifies whether the request concerns a joining or a leaving action for the listed sessions;

- the field session_identifier $n$ gives the identification of the multicast session $\left(S_{i}, G_{j}\right)$, defined by a source $S_{i}$ and group $G_{j}$.

This message is sent by the link layer of each RCST that receives a new PIM-SM join/prune from one of its terrestrial interfaces 
to the NCC only if the switching_label of the corresponding session (if it exists) in its switching table does not contain the spot to which it belongs.

\section{Handling PIM-SM Messages}

In this section, we present the different operations that the system entities have to do when receiving PIM-SM signaling messages. Depending on the routing approach (label-switching or self-switching) used and other factors, the RCSTs, the satellite, and the NCC, handle these messages differently. In the following sections, we detail the processing of these messages.

1) Sending PIM-SM Join Messages: When an RCST receives a PIM-SM join message from its terrestrial interface, it first checks whether the corresponding session is active or not by consulting its subscription table. If it is not the case, the RCST simply ignores the message. In contrast, if the session is active, it verifies if the session is already broadcasted to the spot beam which it belongs to and by checking the switching_label value. If it is the first RCST in its spot beam which joins the session, it informs the NCC, that it is interested by this session. In other words, this RCST receiver sends a DULM join/leave session message (see Fig. 11) with $\mathrm{J} / \mathrm{L}$ bit set to zero, to the NCC in order to update the switching_label value in its MST. As explained in Section VII-A, the NCC broadcasts the new session_descriptor of the corresponding session to the RCSTs.

For the label-switching approach, the same descriptor is also sent to the satellite through a specific input port in order to be able to update its switching table (see Table II), which provides for each session_identifier, the switching_label value.

The RCSTs that receives the broadcasted message from the NCC, should update the entry of the corresponding session in their subscription tables. When an RCST detects that the session being announced is already declared in its mapping table, it concludes that the source is behind him and allows further multicast packets of this session to be sent to the satellite interface. At the same time, it updates the switching_label value in its mapping table when using the switching label approach. Recall that for the label-switching approach, in each MPEG2 packet, the RCST specifies only the session_identifier, while for the self-switching approach, it must also specify the switching_label for each packet.

2) Receiving PIM-SM Join Messages: An IGMP membership report [1] is sent by an end-host (the receiver) to its designated router (DR) in order to join one or more multicast sessions. When receiving this message, the receiver's DR, sends a PIM-SM join including the list of sessions to be joined, to the rendezvous point (RP). We consider the case when the path toward the RP include the satellite segment. In other words, there is an RCST that will receive the multicast packets from the satellite and forward them to the end-host.

When receiving a PIM-SM join message, this RCST consults its subscription table (see Table IV) and the action to do depends on the value of the state of each session that the downstream end-host wants to join.

If the state is active, then it modifies it to online and it starts to take into account the corresponding session_identifier. In other

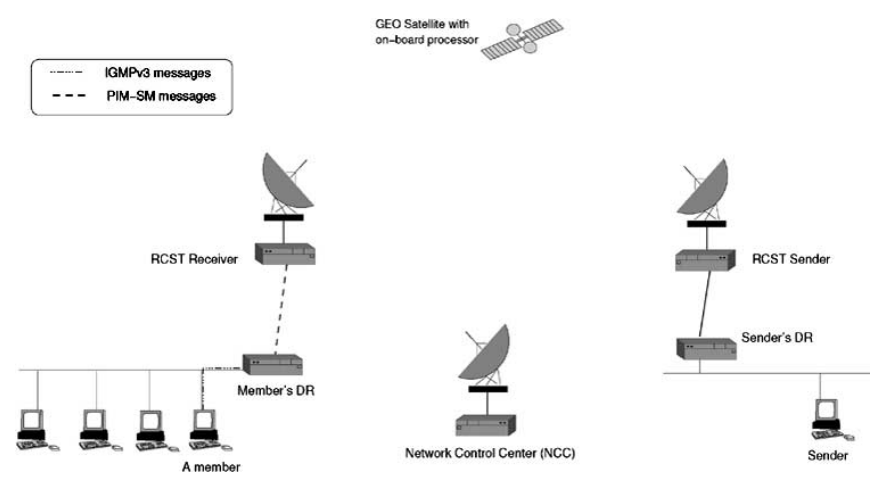

Fig. 12. Transmission of the PIM-SM join message.

words, it will not forward the PIM-SM join message to the RP because it is already receiving the session data from the satellite segment given that there is at least one another RCST which belongs to the same spot beam who is subscribed to the same session. It has only to accept the MPEG data segments at the link layer and forward them to the IP-optimized encapsulation layer.

If the state is standby or available (which means that there may be other members but they belong to other spot beams), the RCST sends to the NCC a DULM join/leave message (see Fig. 11), and as soon as it receives a confirmation of subscription from the NCC (see Table VIII), ${ }^{8}$ it turns the state to online.

In the case of when the session does not exist in the subscription table, this means that there is no multicast source sending packets to the requested multicast group using the satellite network because there is no PIM-SM join message received by the RP from the satellite segment. In this case, we allow the RCST to forward the received PIM-SM join message toward the RP through the satellite network. The RP will receive this join from another RCST, which will forward after the multicast packets to the members through the satellite link. At the same time, this RCST sender (which is behind the source) sends a DULM join/leave message (see Fig. 11) to the NCC, which will attribute a session_identifier to the new session and creates a new entry in the subscription table. All the bits, except that of the spot beam which belongs to the RCST receiver who has sent the join message of the switching_label value will be set to 0 and the state to online. The NCC broadcasts the corresponding session_descriptor (see Table VIII) to all the RCSTs and the RCST receiver creates a new entry in its subscription table that describes the new multicast session.

3) Receiving PIM-SM Prune Messages: The RCST receiver who receives a PIM-SM Prune from one of its terrestrial interface, should first check if there are other receivers interested by the session reached from other interfaces. ${ }^{9}$ If not, it sends to the NCC a DULM join/leave session message (see Fig. 11) and sets the $\mathrm{J} / \mathrm{L}$ bit to 1 to indicate that it is a Leave message.

\footnotetext{
${ }^{8}$ The NCC will switch the bit of the spot beam of the RCST to 1 in the switching label in order to duplicate the data segments on-board the satellite of this spot beams.

${ }^{9}$ The list of outgoing interfaces is given the oif field of the corresponding session in the corresponding entry in the multicast routing table maintained by PIM-SM.
} 
When the RCST which has just left the session is the last member in the spot-beam, all the system entities should update some information. Note that a mechanism based on the counting of the number of members in a multicast session as that we have proposed in [14] could be used for the new-generation of GEO satellite systems to detect when the last member has left the session.

The NCC updates the switching_label field of the pruned session by setting the corresponding bit to zero, so as the packet will not any more be forwarded to this spot beam.

When using the label-switching approach, the RCST sender will be concerned by this change as long as the switching_label is different to $0 \times 0000$, and they should stop sending data to the satellite link. In the case of using the self-switching approach, they must update the switching_label field of packets belonging to the corresponding session (see Table VI) in order to avoid that the satellite forward the multicast to the spot being removed from the list of destination spots.

For the satellite, in the case of the label-switching, it must update its switching table (see Table VI), while for the selfrouting approach, there is nothing to change.

\section{CONCLUSION}

Two different approaches that provide an efficient multicast delivery in the next generation of satellite systems have been proposed in this first part of this paper. The label-switching approach is based on maintaining a switching table on board the satellite, while the self-switching approach includes from a label in each data segment to allow the on-board satellite processor to switch it to the corresponding outgoing ports (spot beams).

In the second part of this paper, we described a new protocol called SMAP, which is an adaptation of PIM-SM for the the new generation of GEO satellite networks.

Within the exciting research area of the multicast delivery over the next generation of GEO satellite systems, there are several future works that could be investigated as an extension of our work described in this paper. One possible extension is the comparison of both approaches using real implementation using real test-bed networks. Another area of research work that could also be investigated, is how to implement both approaches in the satellite systems and to switch between both approaches depending on the expected complexity and the satellite operator requirements.

\section{REFERENCES}

[1] B. Cain, S. Deering, I. Kouvelas, and A. Thyagarajan, Internet group management protocol: Version 3, May 2002. IETF, Internet Draft, draftietf-idmr-igmp-v3-11.txt.

[2] H. D. Clausen, H. Linder, and B. Collini-Nocker, "Internet over direct broadcast satellites," IEEE Commun. Mag., pp. 146-151, June 1999.

[3] H. D. Clausen, H. Linder, and G. Fairhurst, Simple encapsulation for transmission of IP datagrams over MPEG-2/DVB networks, Apr. 2002. IETF, Internet Draft, draft-unisal-ipdvb-enc-00.txt.

[4] S. Deering, "Host extensions to IP multicasting," IETF, RFC 1112, May 1990.

[5] Digital Video Broadcasting (DVB): Interaction Channel for Satellite Distribution Systems, European Telecommunication Standards Institute (ETSI), DVB-RCS001rev14, Apr. 2000.
[6] Digital Video Broadcasting (DVB); DVB Specification for Data Broadcasting, European Telecommunication Standards Institute (ETSI), EN 301192 v1.2.1, June 1999

[7] Digital Video Broadcasting (DVB), Specification for Service Information (SI) in DVB Systems, European Telecommunication Standards Institute (ETSI), EN 300468 v1.4.1, Nov. 2000.

[8] Digital Video Broadcasting (DVB): Framing Structure, Channel Coding and Modulation for 11/12 GHz Satellite Services, European Telecommunication Standards Institute (ETSI), EN 300421 v1.1.2, Aug. 1997.

[9] Digital Video Broadcasting (DVB); Guidelines on Implementation and Usage of Service Information (SI), European Telecommunication Standards Institute (ETSI), ETR 211, Aug. 1997.

[10] Digital Broadcasting Systems for Television, Sound and Data Services; Allocation of Service Information (SI) Codes for Digital Video Broadcasting (DVB) Systems, European Telecommunication Standards Institute (ETSI), ETR 162, Oct. 1995.

[11] Digital Video Broadcasting (DVB); Implementation Guidelines for the Use of MPEG-2 Systems, Video an Audio in Satellite, Cable and Terrestrial Broadcasting Applications, European Telecommunication Standards Institute (ETSI), ETR 154, Sept. 1997.

[12] Multiprotocol Encapsulation, European Telecommunication Standards Institute (ETSI), Draft EN 301192 V1.1.1, 1997-08.

[13] G. Fairhurst, H. D. Clausen, B. Collini-Nocker, and H. Linder, Requirements for Transmission of IP Datagrams Over DVB Networks, May 2002. IETF, Internet draft, draft-fair-ipdvb-req-01.txt.

[14] F. Filali, H. Asaeda, and W. Dabbous, "Counting the number of members in multicast communication," presented at the Int. Workshop Networked Group Communication (NGC 2002), Boston, MA, Oct. 2002.

[15] H. Holbrook and B. Cain, Source-specific multicast for IP, Mar. 2000. IETF, Internet Draft, draft-holbrook-ssm-00.txt.

[16] B. Fenner, M. Handley, H. Holbrook, and I. Kouvelas, Protocol Independent Multicast-Sparse Mode (PIM-SM): Protocol Specification (Revised), IETF, Nov. 2001. draft-ietf-pim-sm-v2-new-04.ps.

[17] B. G. Haskell, A. Puri, and A. N. Netravali, Digital Video: An Introduction to MPEG-2. London, U.K.: Chapman \& Hall, 1997, vol. 1.

[18] Information Technology_Generic Coding of Moving Pictures and Associated Audio: Systems, ISO/IEC 138 18-1, Nov. 1994.

[19] Information Technology-Generic Coding of Moving Pictures and Associated Audio Information: Part 6, Extension for Digital Storage Media Command and Control, ISO/IEC 13 818-6, 1998.

[20] Information Technology_Generic Coding of Moving Pictures and Associated Audio Information. Part 1: Systems, ISO/IEC 13818-1, Nov. 1994.

[21] N. K. G. Samaraweera, "Return link optimization for Internet service provision using DVB-S networks," in Proc. SIGCOMM Computer Communication Review, vol. 29, July 1999, pp. 4-13.

[22] IP Multicast for Digital MPEG Networks, Society of Cable Telecommunications Engineers, Engeneering Committee, Digital Video Subcommittee, DVS 311, 2001.

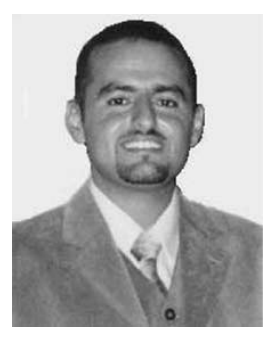

Fethi Filali received the computer science engineering and DEA degrees from the National College of Informatics (ENSI), Tunisia, in 1998 and 1999, respectively, and the Ph.D. degree in computer science from the Université de Nice Sophia-Antipolis (UNSA) which he has conducted in the Planète research team of the Institut National de Recherche en Informatique et Antomatique (INRIA) of Sophia-Antipolis.

During 2003, he was a non permanent Teaching and Research Assistant with the Université de Nice Sophia-Antipolis. He is with the Mobile Communications Department, Eurécom Institute, Sophia-Antipolis, France, as an Assistant Professor, since September 2003. His current research interests include routing and TCP performance in mobile ad hoc networks, QoS support in IEEE 802.11 based networks, multimedia applications transport in wireless networks. 


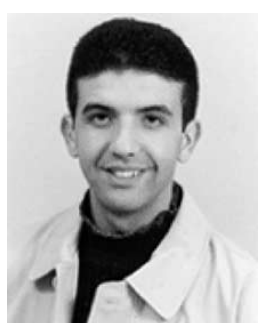

Ghassane Aniba received the Dipl.-Ing. degree in telecommunication engineering from the Institut National des Postes et Telecommunications (INPT), Rabat, Morocco, in 2002. He is currently working toward the Ph.D. degree at the Institut National de la Recherche Scientifique, Energie, Matériaux et Télécommunications (INRS-EMT), Montreal, QC, Canada.

For his Ing. degree, he worked on the adaptation of multicast protocols over satellite link (IP over DVB), with the Planète Reseach Team at the Institut National de Recherche en Informatique et Automatique (INRIA), Sophia Antipolis, France. His research interests include traffic modeling in wireless networks, MIMO systems in HSDPA, and QoS in the third generation of wireless networks.

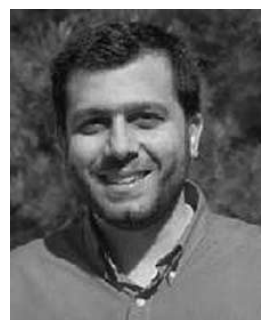

Walid Dabbous received the electrical engineering degree from the Faculty of Engineering, Lebanese University, Beirut, Lebanon, in 1986, and the DEA and Doctorat d'Université degrees from the University of Paris XI, Orsay, France, in 1987 and 1991, respectively.

He joined the RODEO Team at the Institut National de Recherche en Informatique et Automatique (INRIA), Sophia Antipolis, France, in 1987. Since 1991, he has been a Staff Researcher at INRIA and Leader of the Planète team since 2001. His main research topics are high-performance communication protocols, congestion control, reliable multicast protocols, audio and video conferencing over the Internet, efficient and flexible protocol architecture design, and the integration of new transmission media such as satellite links in the Internet. 